hep-th/0604046

\title{
Scaling solutions and geodesics in moduli space
}

\author{
J.L.P.Karthauser ${ }^{1 \S}$, P.M.Saffin ${ }^{1,2}$ ฯ \\ ${ }^{1}$ Department of Physics and Astronomy, University of Sussex \\ Falmer, Brighton BN1 9QJ, UK \\ ${ }^{2}$ School of Physics and Astronomy, University of Nottingham \\ University Park, Nottingham NG7 2RD, UK
}

\begin{abstract}
In this paper we consider cosmological scaling solutions in general relativity coupled to scalar fields with a non-trivial moduli space metric. We discover that the scaling property of the cosmology is synonymous with the scalar fields tracing out a particular class of geodesics in moduli space - those which are constructed as integral curves of the gradient of the log of the potential. Given a generic scalar potential we explicitly construct a moduli metric that allows scaling solutions, and we show the converse - how one can construct a potential that allows scaling once the moduli metric is known.
\end{abstract}

\footnotetext{
§email: jlk23@sussex.ac.uk

『email: paul.saffin@nottingham.ac.uk
} 


\section{Introduction}

Scaling solutions to the Friedman equation are of prime interest in modern cosmology, both for study of early time inflationary scenarios and, more recently, the late time accelerating expansion of the universe which is suspected to be driven by some dark energy component in the cosmological fluid [1, 2]. The utility of these scaling regimes is that the various components can evolve such that a constant ratio is maintained between their energy densities, and as attractor solutions they approach these ratios without fine tuning of the initial conditions and so can be used to explain the cosmic coincidence of energy densities [3, 4. Scaling solutions also find a use in the earlier epoch of inflation, where exponential potentials [5, 6, 7] allow for exact solutions and sums of exponentials can aid inflationary behaviour [8]. The dynamics of scalar fields with such potentials is now well understood, and is neatly expressed using ideas from dynamical systems for single scalars [9] and multiple scalars [10, 11, 12.

That scalar field models can be constructed with potentials that can mimic the conditions we observe in the universe is but one part of the story. The theories proposed require a finetuning to match observation, and we are still as of yet to detect the direct signature of scalar fields in nature; is there any fundamental physics that might motivate their existence? Fortunately scalar fields abound in the low energy limit of many modern particle physics models where they appear dynamically in the form of moduli which parametrize the size and shape of the geometry of internal dimensions. Interestingly in many of these models, particularly unified gravity models with dimensional reduction, gaugino condensation or instanton corrections, the low energy effective potentials are often exponential in form 13, 14, 15, 16, 17, 18, 19, 20, 21, 22. It would be intriguing indeed if the scalar potentials used in inflationary theory and quintessence were in fact a consequence of dynamics of some extra dimensional string or M-theory.

However, exponential potentials are but one class of possible potential. How might we investigate the scaling behaviour of more general potentials? As cosmologists we are well aware of the geometric nature of pure gravity in Einstein's equations, however when we couple in additional scalar fields they also come with their own geometry. As the system evolves one can think of the kinetic term providing a measure of distance in field space. This is by virtue of the existence of a moduli space metric, which for minimal kinetic terms is flat, $\partial_{\mu} \phi^{a} \partial^{\mu} \phi^{a} \equiv \delta_{a b} \partial_{\mu} \phi^{a} \partial^{\mu} \phi^{b}$, but there is no reason a priori not to also consider other forms for this metric too, perhaps providing a useful geometric insight into the dynamics of the system.

There is already a tradition of the study of moduli space metrics within particle physics models, particularly in supersymmetric field theories where the field space geometry is usually of some special type, for example Kähler geometry. More recently Townsend and Wohlfarth proposed thinking of cosmological evolution in an augmented field space [23], where the scale factor is added to the geometry of the field space. It is then discovered that cosmological solutions can be thought of as geodesics on this extended manifold. Here however we do not consider this augmented space, rather we look at the field moduli space alone and discover that in a scaling regime the fields trace out a particular type of geodesic, namely a geodesic which coincides with an integral curve of $\partial_{a} \ln (V)$. Given that the moduli metric, $G_{a b}$, and the potential, $V$, are in principle independent there may be no such geodesic, or indeed there may be many congruences of such geodesics. Here we explicitly construct a $G_{a b}$ (although there may also be others) that allows scaling solutions with a generic potential and we also show how one can find a potential that allows scaling if $G_{a b}$ is known. 


\section{Scaling Cosmology and Geodesics}

The starting point for this work is a simple observation following from previous studies of multi-field systems with $G_{a b}=\delta_{a b}$. It has been shown [10, 11, 12] that the scaling regime is characterized by

$$
\begin{aligned}
\dot{\phi}^{a} & =\frac{A^{a}}{t}, \\
\Rightarrow \phi^{a} & =A^{a} \ln (t)+B^{a} .
\end{aligned}
$$

So given two different fields we have

$$
\phi^{a}=\left(\frac{A^{a}}{A^{b}}\right) \phi^{b}+\left(B^{a}-\frac{A^{a}}{A^{b}} B^{b}\right),
$$

which is just the equation of a straight line. As the moduli metric is flat for these cases then this straight line is, of course, a geodesic. It is our aim to show that even for $G_{a b} \neq \delta_{a b}$ this relation between scaling and geodesics persists.

We start by describing the system we aim to study, namely a set of scalar fields with moduli metric $G_{a b}$ and potential $V$ coupled to gravity. The Lagrangian is given by

$$
\mathcal{L}=\sqrt{-g}\left[\frac{1}{2 \kappa^{2}} \mathcal{R}-\frac{1}{2} G_{a b} \partial_{\mu} \phi^{a} \partial^{\mu} \phi^{b}-V(\phi)\right]
$$

with the indices $a, b, c, \ldots \in\{1,2, \ldots, n\}$ running over the number of scalar fields. We adopt a conventional Friedman-Robertson-Walker cosmology ${ }^{1}$ for the space-time metric and restrict the scalar to vary only with time. This then allows us to write down the effective Lagrangian for the reduced system, where the dynamical variables depend only on time,

$$
\mathcal{L}=a^{3}\left(\frac{1}{2} G_{a b} \dot{\phi}^{a} \dot{\phi}^{b}-V(\phi)\right)-\frac{3}{\kappa^{2}} a \dot{a}^{2} .
$$

Here $a=a(t)$ is the scale factor of the universe, and ${ }^{\cdot} \equiv \partial_{t}=\frac{\partial}{\partial t}$ denotes the derivative with respect to time. In this language we find that the equations of motion, along with the vanishing of the Hamiltonian due to invariance under time reparametrization, are

$$
\begin{aligned}
H^{2} & =\frac{\kappa^{2}}{3}\left(\frac{1}{2} G_{a b} \dot{\phi}^{a} \dot{\phi}^{b}+V\right), \\
\dot{H}+3 H^{2} & =\kappa^{2} V, \\
\ddot{\phi}^{a}+\Gamma_{b c}^{a} \dot{\phi}^{b} \dot{\phi}^{c}+3 H \dot{\phi}^{a} & =-G^{a b} \partial_{b} V,
\end{aligned}
$$

with the Hubble parameter $H \equiv \frac{\dot{a}}{a}$ and the affine connection $\Gamma_{b c}^{a}$ of $G_{a b}$ defined in the conventional way,

$$
\Gamma_{b c}^{a}=\frac{1}{2} G^{a d}\left(G_{b d, c}+G_{c d, b}-G_{b c, d}\right) .
$$

Given that we shall be discussing geodesics we now give the standard expressions for a geodesic curve, $\mathcal{C}$, with tangent vector $T$ that is parametrized by an affine parameter $\lambda$.

$$
\begin{aligned}
T^{a} & =\frac{d}{d \lambda} \phi^{a}, \\
T^{a} \nabla_{a} T^{b} & =0 \Rightarrow \phi^{\prime \prime a}+\Gamma_{b c}^{a} \phi^{\prime b} \phi^{\prime c}=0, \\
T^{a} T_{a} & =1,
\end{aligned}
$$

\footnotetext{
${ }^{1}$ In this paper we take a space-time metric of signature $(-+++)$.
} 
where ${ }^{\prime} \equiv \frac{d}{d \lambda}$.

We shall take scaling to mean that the terms in (2.6) and (2.7) evolve in constant proportion to one another, $H^{2} \propto \dot{H} \propto V \propto G_{a b} \dot{\phi}^{a} \dot{\phi}^{b}$. We start by considering $H^{2}$ and the kinetic term of (2.6) and enforce proportionality by introducing a constant $\alpha$,

$$
\alpha^{2} H^{2}=G_{a b} \dot{\phi}^{a} \dot{\phi}^{b}
$$

We now want to see if this can be consistent with $\phi^{a}(t)$ tracing out a geodesic, meaning that the affine parameter can now be thought of as a function of cosmic time $\lambda=\lambda(t)$. The normalisation of the tangent vector (2.12) then gives us

$$
G_{a b} \dot{\phi}^{a} \dot{\phi}^{b}=\left(\frac{d \lambda}{d t}\right)^{2}
$$

which combines with (2.13) to produce

$$
a=a_{0} \exp (\lambda / \alpha)
$$

Converting time derivatives into $\lambda$ derivatives and using (2.7) along with (2.11) one finds that (2.8) becomes

$$
\phi^{\prime a}=-\frac{1}{\alpha \kappa^{2}} G^{a b} \partial_{b} \ln (V)
$$

This is very suggestive, and to make manifest its relation to geodesics more explicit we shall write it as the components of the tangent co-vector,

$$
T_{a}=\partial_{a} W, \quad W=-\frac{1}{\alpha \kappa^{2}} \ln (V) .
$$

So, if our scaling/geodesic ansatz is consistent with the other terms in the equations we see that the scaling geodesic takes a very particular form, it is the integral curve of $\nabla W$. An immediate consequence of this is that one can never get a scaling solution on closed geodesics.

We also need to check that the potential evolves in constant proportion to $H^{2}$, which we can do by using the fact that these tangent vectors have been normalized to unity,

$$
\phi^{\prime a}\left(-\frac{1}{\alpha \kappa^{2}}\right) \partial_{a} \ln (V)=1
$$

which is integrated to give

$$
V=V_{0} \exp \left(-\alpha \kappa^{2} \lambda\right)=V_{0}\left(\frac{a}{a_{0}}\right)^{-\alpha^{2} \kappa^{2}} .
$$

If it is the case that $V \propto H^{2}$ then we can integrate to find

$$
a^{\alpha^{2} \kappa^{2} / 2} \propto\left(t-t_{0}\right)
$$

which, when substituted back into the potential (2.19), gives the conclusion that

$$
V \propto \frac{1}{t^{2}}
$$


This is precisely the form that $V$ must take as is easily seen by inspecting (2.6) (2.7). We also note from (2.20) that we get an accelerating scale factor if $\alpha \kappa<\sqrt{2}$. We can understand such a requirement by reconsidering (2.12) and (2.17) and writing them in the form

$$
\frac{1}{2 \kappa^{2}} G^{a b} \partial_{a} V \partial_{b} V / V^{2}=\frac{1}{2} \alpha^{2} \kappa^{2}
$$

and now we recognize the left hand side to be the multi-field generalisation of the slow-roll parameter $\epsilon$ [24]. We should point out that the potential is only required to take the form (2.19) on the geodesic itself, away from the geodesic the potential can take any form, or even be undefined.

A brief comment is in order about the nature of $\alpha$. We see from (2.6) and (2.13) that

$$
\begin{aligned}
\tilde{T} & :=\frac{1}{2} \frac{G_{a b} \dot{\phi}^{a} \dot{\phi}^{b}}{H^{2}}=\frac{1}{2} \alpha^{2}, \\
\tilde{V} & :=\frac{V}{H^{2}}=\frac{3}{\kappa^{2}}-\frac{1}{2} \alpha^{2},
\end{aligned}
$$

and so it seems in scaling solutions $\alpha$ determines the value of both the kinetic and potential energies. Moreover, if the potential is positive then we have an upper bound on $\alpha$ given by $\alpha \kappa<\sqrt{6}$.

\section{Construction of a field space metric}

We now come to the issue of whether we can construct a metric $G_{a b}$ that will allow scaling for a given potential $V$. Using (2.17) along with (2.11) we find that this amounts to solving

$$
G^{a b} \partial_{a} W\left(\partial_{b} \partial_{c} W-\Gamma_{b c}^{d} \partial_{d} W\right)=0,
$$

for the metric. At first sight this looks a difficult task, and a general solution probably is out of reach. However, it is possible to solve this equation if we restrict ourselves to a conformally flat metric

$$
d s^{2}=\exp (2 \Omega) d \vec{\phi}^{2}
$$

with connection components

$$
\Gamma_{b c}^{a}=\delta_{b}^{a} \Omega,_{c}+\delta_{c}^{a} \Omega,_{b}-\delta_{b c} \delta^{a d} \Omega,_{d} .
$$

This is a natural choice in that (3.25) is now an equation for a single scalar function $\Omega(\phi)$ in terms of the single scalar function $V(\phi)$. Given that we have already normalized the tangent vector in (2.12) this immediately gives us the conformal factor

$$
\exp (2 \Omega)=\sum_{a} \partial_{a} W \partial_{a} W=\frac{1}{\alpha^{2} \kappa^{4}} \sum_{a} \frac{\partial_{a} V \partial_{a} V}{V^{2}}
$$

which, as is easily checked, does indeed satisfy (3.25). One consequence of this solution is that if $V$ goes through zero with non-vanishing gradient then the conformal factor diverges there, pushing such points to an infinite proper distance in moduli space. Hence in a scaling solution $V$ will not change sign. Another important aspect of this solution is that it allows for a scaling solution through each point in field space where $\ln (V)$ is well defined; it does not restrict to a single path as is the case for the usual multi-field scaling solution. 
The conformally-flat solution we have presented is not necessarily the only metric on field space to give scaling solutions, however we leave investigation of the construction of more general metrics for future work.

The other way to proceed is to find a potential that yields a scaling solution for a given metric. We do not have an explicit solution to this problem but one certainly exists. Once a moduli metric has been chosen one finds a suitable, i.e. not closed, geodesic $\mathcal{C}$. Using the affine parameter of this geodesic one can reconstruct the value of the potential along $\mathcal{C}$ using (2.19). Simply having $V$ along the geodesic is not enough to keep $\phi^{a}$ on the scaling solution, we also need $\partial_{a} V$ which we can find from (2.17) as we know the geodesic's tangent vector. This is rather like a race track with banked corners to keep the cars from coming off - the gradient of $V$ keeps the scalars on the scaling solution.

\section{Constructing scaling solutions}

Now we have set up the framework for finding scaling solutions in general potentials we shall give some examples to show how the process works, by numerically evolving (2.6) (2.7) (2.8). We shall start with a rather simple example, reminiscent of the multi-field scaling solutions which required exponential potentials,

$$
V=\exp \left(\phi^{1}\right)+\exp \left(2 \phi^{2}\right)
$$

using the values $\kappa^{2}=1$ and $\alpha=1$. In Fig. 1 we have plotted the evolution of $\phi^{1}$ and $\phi^{2}$, also showing the vector field $\partial_{a} \ln V$ (the arrow lengths have been normalized for clarity). The solid lines denote scaling solutions and we note that the fields do indeed follow the gradient of $\ln (V)$ as

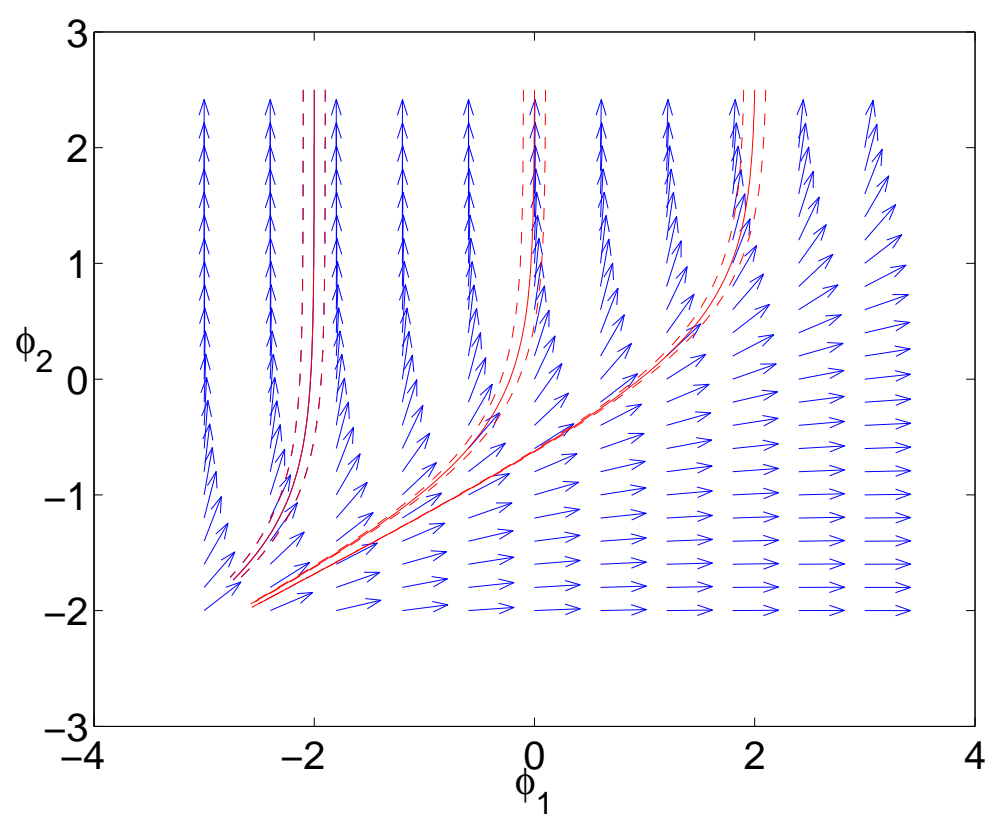

Figure 1: This plot shows the vector field $\nabla \ln V$ of the potential (4.29), along with some example field evolutions. The solid lines are the scaling solutions, with the dashed lines being perturbations about the scaling solution. 

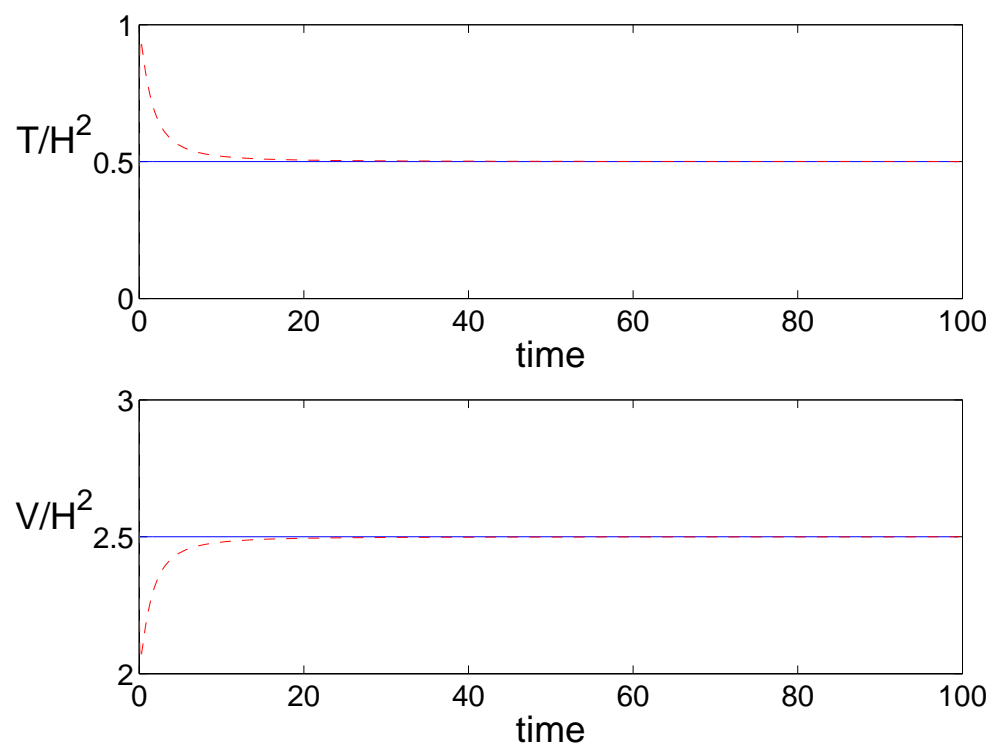

Figure 2: This plot shows the ratio of the kinetic, potential energy to $H^{2}$ for a scaling solution and perturbations around it.

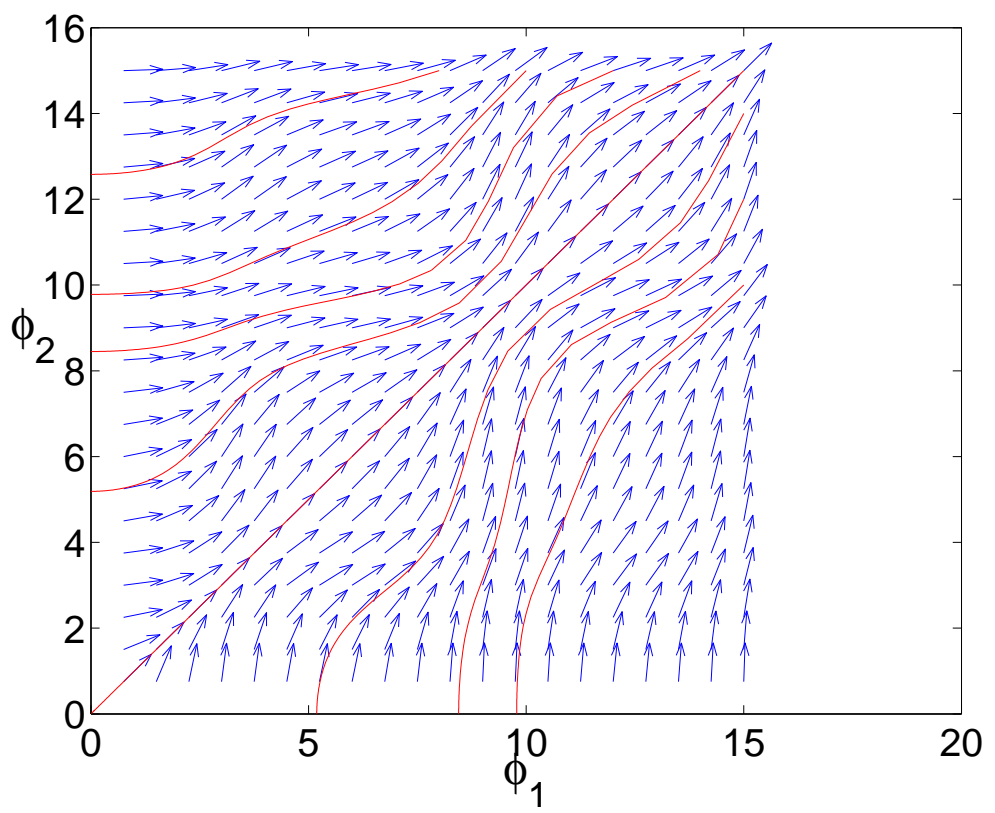

Figure 3: This is the gradient of the potential (4.30), along with the scaling solutions. 
expected. The dashed lines represent perturbations of these initial conditions, and we see that they do not simply go back to the scaling solutions of the solid lines. This however does not mean that scaling solutions are not attractors as Fig. 2 makes clear. Fig. 2 is a plot showing the values of the kinetic and potential energies for a scaling solution (solid line) and a perturbation of that solution (dashed line). What we see is that the perturbed case does approach a scaling solution, just not the one you started with. This is because when using the conformally flat moduli metric to solve (3.25) there is a scaling solution through each point in moduli space. Note also that $T / H^{2}, V / H^{2}$ approach the values as predicted by (2.23) and (2.24).

As a further test let us consider a more involved, bumpy, potential,

$$
V=\left(2+\sin \left(\phi^{1}\right)\right)\left(2+\sin \left(\phi^{2}\right)\right) \text {. }
$$

Scaling solutions for this can be seen in Fig. 3 also following integral curves of the gradient of $\ln (V)$, as our analysis shows they should.

\section{$5 \quad$ Adding a barotropic fluid}

The effects of adding a baratropic fluid to the system can be studied by extending our Friedman equation (2.6) and adding the fluid equations,

$$
\begin{aligned}
H^{2} & =\frac{\kappa^{2}}{3}\left(\frac{1}{2} G_{a b} \dot{\phi}^{a} \dot{\phi}^{b}+V+\rho\right), \\
P & =(\gamma-1) \rho, \\
\dot{\rho} & =-3 \gamma H \rho,
\end{aligned}
$$

where $P$ is the fluid pressure and $\rho$ is its density. Such a fluid is typically invoked to represent species of particles in the early Universe other than the scalar field and so constitutes an important addition.

Although we have not thoroughly explored the consequences of adding such a fluid to the system we can find a bound on the equation of state parameter, $\gamma$, which reduces to the standard one of the usual scaling analysis. The analysis follows the same lines as the one presented above, using the scaling ansatz (2.13) as well as

$$
V+(1-\gamma / 2) \rho=\beta V
$$

and taking $\beta$ as a new parameter which is constant during scaling. With this addition we find that (2.16) is altered to become

$$
\phi^{\prime a}=-\frac{1}{\alpha \beta \kappa^{2}} G^{a b} \partial_{b} \ln (V) .
$$

A consequence of (5.34) is that if the fluid has $\gamma<2$, which is physically reasonable, then for positive $V$ and $\rho$ we require $\beta>1$. This is the origin of the bound we shall derive.

In much the same way that (2.19) is derived, one finds that

$$
\rho \propto a^{-\alpha^{2} \beta \kappa^{2}},
$$

however we also know by integrating (5.33) that $\rho \propto a^{-3 \gamma}$ which gives us

$$
\beta=3 \gamma /\left(\alpha^{2} \kappa^{2}\right)
$$


and so our bound on $\beta$ now becomes a bound on the equation of state parameter

$$
3 \gamma>\alpha^{2} \kappa^{2}
$$

This bound is similar to the one usually found in scaling analyses with a fluid, indeed taking the case of a single scalar with potential $V=V_{0} \exp (b \kappa \phi)$ one has that $\alpha^{2} \kappa^{2}=9 \gamma^{2} / b^{2}$, so (5.38) becomes $b^{2}>3 \gamma$ matching the result found in 9 .

\section{Comments on fixed point analyses}

No paper on scaling dynamics appears to be complete without consideration of the critical point analysis, obtained by recasting the equations of motion into an autonomous systems framework [9. 11. In this approach the asymptotic behaviour and stability of the system can be discovered by analysing the nature of fixed point solutions.

The now standard approach for a system with potential

$$
V=\sum \Lambda_{i} \exp \left(-\vec{\alpha}_{i} \cdot \vec{\phi}\right)=\sum V_{i}
$$

is to define two new sets of variables

$$
\begin{aligned}
X^{a} & \sim \dot{\phi}^{a} / H \\
Y_{i} & \sim V_{i} / H,
\end{aligned}
$$

with which to describe the degrees of freedom. These are useful because in the case of exponential potentials they are constant in the scaling regime, giving scaling solutions as fixed points of the autonomous system. For the more general case we have been discussing the closest analogue would be the term

$$
X \sim \sqrt{G_{a b} \dot{\phi}^{a} \dot{\phi}^{b}} / H
$$

which we know is a constant during scaling. However, this is just one term and we need to describe $n$ momenta $\dot{\phi}^{a}$. In the case where one uses the conformally flat solution of (3.25) one could introduce

$$
X^{a} \sim e^{\Omega} \dot{\phi}^{a} / H
$$

as a natural set of variables. However, the problem reappears when we try to find an analogue for the $Y_{i}$. In general the potential will not split up nicely into a sum of terms, making the analysis problematic. One possible approach to constructing more variables is to use the derivative of the potential, this is a problem which we hope to return to.

\section{Concluding remarks}

In this paper we studied the scaling solutions of a Friedman-Robertson-Walker cosmology containing scalar fields evolving in an arbitrary potential. The standard picture with canonical kinetic terms would lead us to conclude that the potential must take a special form, that of a sum of exponentials, if scaling is to take place. What we have been able to show is that by including the freedom of a metric on moduli space we can recover scaling solutions in generic potentials, with the connection between moduli space geometry and scalar potential being that the scaling geodesics lie along integral curves of $\nabla \ln V$. In fact we have been able to provide a special solution to the scaling 
criteria by way of a conformally flat moduli metric, and we provided explicit examples to show the system in action. The statement also works in reverse; given a metric on moduli space it is possible to construct a potential which will support scaling solutions. We also briefly considered the effects of adding a baratropic fluid into the system and were able to find a bound for the equation of state parameter, with this bound reducing to the standard one when the moduli metric is flat.

There are a number of things which have not been fully addressed is this article. Firstly is the issue of stability. While we saw numerical evidence in our examples that the system approached a scaling solution a more thorough analysis is required, and we expect it will combine some aspects of the moduli geometry along with the potential. An approach that could help with the stability analysis is that of recasting the system in a form inspired by dynamical systems, writing the equations as an autonomous system. While we have not presented such a framework we believe this approach is well worth studying.

Acknowledgements The authors would like to gratefully acknowledge useful discussions with Mark Hindmarsh and Harvey Reall. Both JLPK and PMS are supported by PPARC.

\section{References}

[1] T. Padmanabhan, "Dark Energy: Mystery of the Millennium," arXiv:astro-ph/0603114.

[2] E. J. Copeland, M. Sami and S. Tsujikawa, arXiv:hep-th/0603057.

[3] I. Zlatev, L. M. Wang and P. J. Steinhardt, Phys. Rev. Lett. 82, 896 (1999) arXiv:astro-ph/9807002.

[4] L. M. Wang, R. R. Caldwell, J. P. Ostriker and P. J. Steinhardt, "Cosmic Concordance and Quintessence," Astrophys. J. 530 (2000) 17 arXiv:astro-ph/9901388.

[5] J. J. Halliwell, Phys. Lett. B 185, 341 (1987).

[6] C. Wetterich, Nucl. Phys. B 302, 668 (1988).

[7] A. B. Burd and J. D. Barrow, Nucl. Phys. B 308, 929 (1988).

[8] A. R. Liddle, A. Mazumdar and F. E. Schunck, "Assisted inflation," Phys. Rev. D 58 (1998) 061301 arXiv:astro-ph/9804177.

[9] E. J. Copeland, A. R. Liddle and D. Wands, "Exponential potentials and cosmological scaling solutions," Phys. Rev. D 57 (1998) 4686 arXiv:gr-qc/9711068.

[10] E. J. Copeland, A. Mazumdar and N. J. Nunes, Phys. Rev. D 60, 083506 (1999) arXiv:astro-ph/9904309.

[11] A. Collinucci, M. Nielsen and T. Van Riet, "Scalar cosmology with multi-exponential potentials," Class. Quant. Grav. 22 (2005) 1269 arXiv:hep-th/0407047.

[12] J. Hartong, A. Ploegh, T. Van Riet and D. B. Westra, "Dynamics of Generalized Assisted Inflation," arXiv:gr-qc/0602077.

[13] B. S. DeWit, "Relativity, Groups And Topology." Les Houches (1963) Gordon and Breach 1964. 
[14] T. Biswas and P. Jaikumar, JHEP 0408, 053 (2004) arXiv:hep-th/0407063.

[15] C. M. Chen, P. M. Ho, I. P. Neupane, N. Ohta and J. E. Wang, JHEP 0310, 058 (2003) arXiv:hep-th/0306291.

[16] I. P. Neupane and D. L. Wiltshire, Phys. Rev. D 72, 083509 (2005) arXiv:hep-th/0504135.

[17] I. P. Neupane and D. L. Wiltshire, Phys. Lett. B 619 (2005) 201 arXiv:hep-th/0502003.

[18] J. L. P. Karthauser and P. M. Saffin, "The dynamics of coset dimensional reduction," arXiv:hep-th/0601230.

[19] J. P. Derendinger, L. E. Ibanez and H. P. Nilles, "On The Low-Energy D = 4, N=1 Supergravity Theory Extracted From The D = 10, N=1 Superstring," Phys. Lett. B 155, 65 (1985).

[20] M. Dine, R. Rohm, N. Seiberg and E. Witten, "Gluino Condensation In Superstring Models," Phys. Lett. B 156 (1985) 55.

[21] E. Witten, "Non-Perturbative Superpotentials In String Theory," Nucl. Phys. B 474, 343 (1996) arXiv:hep-th/9604030.

[22] G. W. Moore, G. Peradze and N. Saulina, "Instabilities in heterotic M-theory induced by open membrane instantons," Nucl. Phys. B 607, 117 (2001) arXiv:hep-th/0012104.

[23] P. K. Townsend and M. N. R. Wohlfarth, "Cosmology as geodesic motion," Class. Quant. Grav. 21 (2004) 5375 arXiv:hep-th/0404241.

[24] C. P. Burgess, J. M. Cline, H. Stoica and F. Quevedo, JHEP 0409, 033 (2004) arXiv:hep-th/0403119. 